\title{
Study on Axial and Radial Heterostructures of Si-Ge and Si-SiGe Nanowires
}

\author{
Ling Pan, Kok-Keong Lew, Joan M. Redwing, Elizabeth C. Dickey \\ Department of Materials Science and Engineering and Materials Research Institute, \\ Pennsylvania State University, University Park, 16802
}

The fabrication of heterostructured semiconductor nanowires (NWs) is of great interest both for fundamental studies of carrier confinement effects as well as nanoscale device development. In previous studies, homogeneous $\mathrm{SiGe}$ alloy NWs have been successfully synthesized [1]. In this study, we have synthesized both axial and radial heterostructures of Si-Ge or Si-SiGe NWs by vapor-liquid-solid (VLS) mechanism out of nanoporous alumina membranes or on Si wafers using $\mathrm{Au}$ as a catalyst and silane $\left(\mathrm{SiH}_{4}\right)$ and/or germane $\left(\mathrm{GeH}_{4}\right)$ as gas precursors. The chemistry and microstructure of the NWs were characterized by JEOL 2010F field-emission TEM / STEM, equipped with energy-dispersive $\mathrm{x}$-ray spectroscopy (EDS).

Si-SiGe axial heterostructured NWs are grown at $500^{\circ} \mathrm{C}$ by periodically switching the source gas between $\mathrm{SiH}_{4}$ and a mixture of $\mathrm{SiH}_{4}$ and $\mathrm{GeH}_{4}$. Fig.1a and $1 \mathrm{~b}$ are bright-field TEM and darkfield STEM images of such a typical NW, where a (111)[11 $\overline{2}]$ twin is clearly seen in the center of the wire. The Si and SiGe segments as well as the Au/Si interface, exhibit faceting at ( $\overline{1} \overline{1} 1)$ surfaces, indicating the $(\overline{1} \overline{1} 1)$ growth front. An EDS line scan is performed along the NW (as indicated by the line in Fig. 1b), and the resulting profile in Fig. 1c shows periodic modulation of the Si (has small concentration of Ge due to the short period between the source gas switching schemes) and SiGe segments.

With additions of pure $\mathrm{GeH}_{4}$ at $500^{\circ} \mathrm{C}$, a temperature much higher than the optimum growth temperature for pure Ge NWs, the decomposition of $\mathrm{GeH}_{4}$ gas takes place so fast that chemical vapor deposition (CVD) of Ge on the NW surfaces dominates leading to a radial heterostructure. Our experiments confirm the formation of three-dimensional island structures around the initially grown Si NW core. Fig. 2 shows bright-field TEM and dark-field STEM images of the crosssection of a $[11 \overline{2}] \mathrm{Si}$ core NW with the surrounding 3-dimentional (3D) Ge islands. In addition, a thin wetting layer of Ge is clearly seen around the Si core in the dark-field STEM image, suggesting the Stranski-Krastanov (S-K) growth mechanism. Faceting on $\{111\},\{110\}$ and $\{113\}$ is clearly seen not only on the outer surface of the Si core, but also on the surfaces of the Ge islands. When a higher $\mathrm{GeH}_{4}$ partial pressure is used in the growth, Si-Ge core-shell structures are obtained, as shown in Fig. 3, where $3 \mathrm{a}$ and $3 \mathrm{~b}$ are the bright-field TEM and darkfield STEM images, and 3c is the EDS line scan profile across the NW. 2D Moiré fringes are frequently observed in these islanded or core-shell NWs, implying the lattice mismatch between the core and the islands or the shell. The interface structure is characterized together with the strain analyses.

\section{References:}

[1] K.-K. Lew et al., Adv. Mater. 15 (2003) 2073.

[2] This work was supported by the NSF under grant DMR-0103068 and The Penn State University MRSEC for Nanoscale Science (DMR-0213623). 

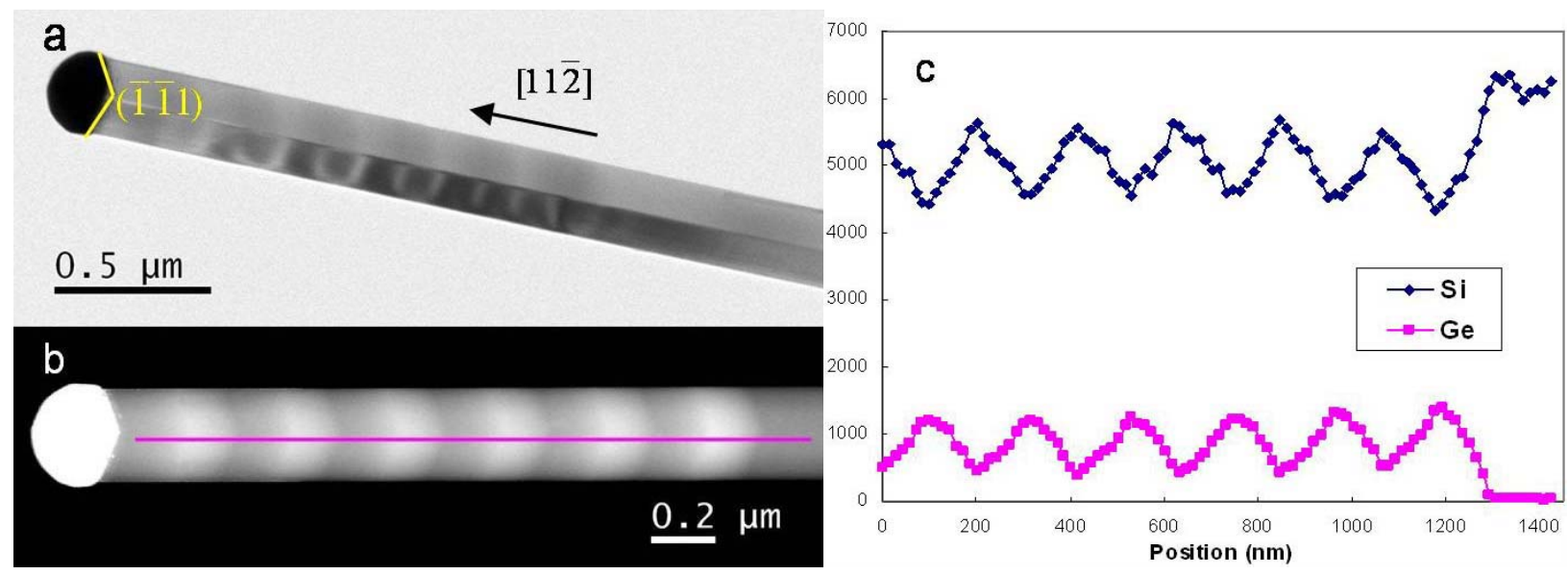

Fig. 1. (a) Bright-field TEM and (b) dark-field STEM images of an axially modulated Si-SiGe nanowire with a (111) $[11 \overline{2}]$ twin in the middle. (c) EDS line scan profile along the line in (b).
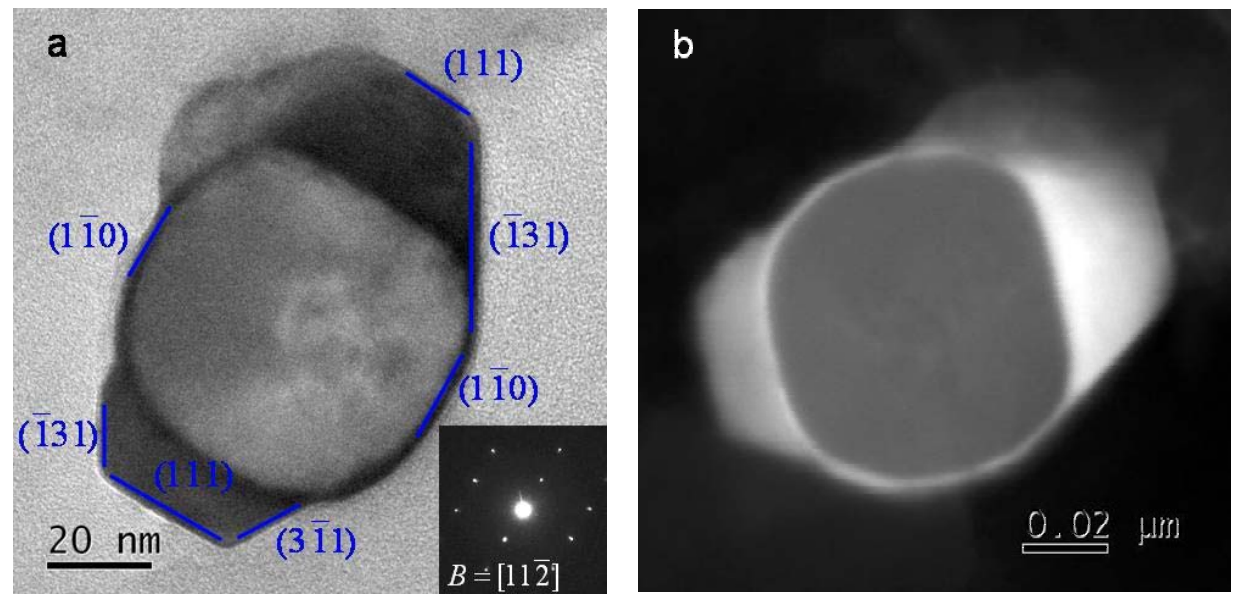

Figure 2. (a) Bright-field TEM and (b) dark-field STEM images of the 3D Ge islands depositing around Si nanowire cores. A thin wetting layer is clearly seen surrounding the core.
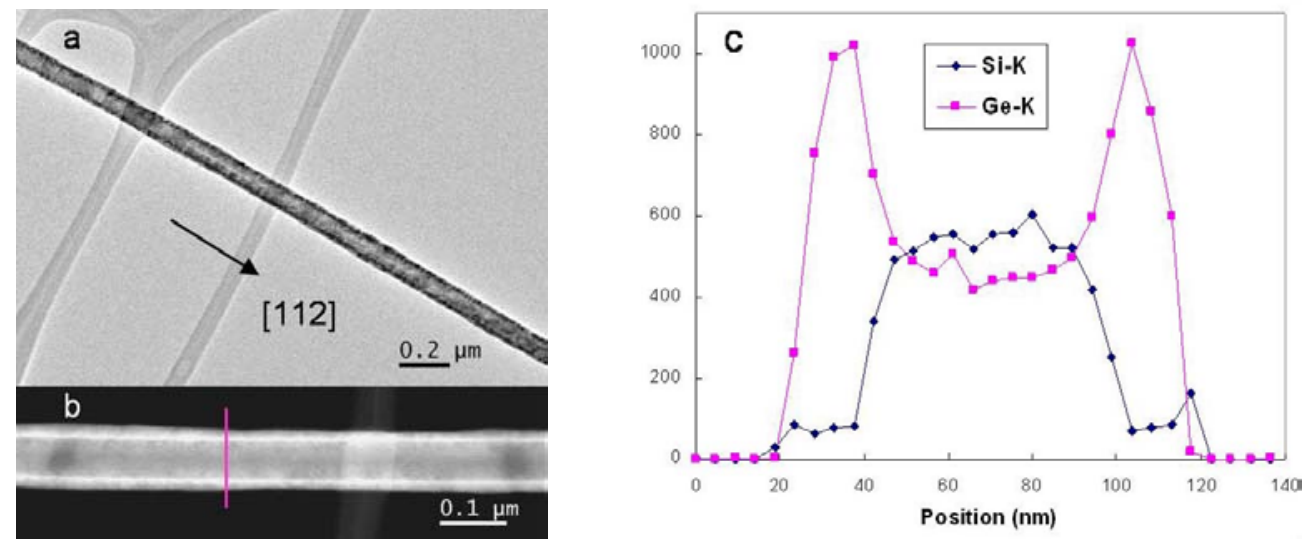

Fig. 3. (a) Bright-field TEM and (b) dark-field STEM images of a core-shell Si-Ge nanowire. (c) EDS line scan profile across the nanowire along the line in (b). 